\title{
Pigmentos Lipossolúveis e Hidrossolúveis em Plantas de SAlvínia sob TOXICIDAde POR CROMO ${ }^{1}$
}

\author{
Liposoluble and Hydrosoluble Pigments in Salvinia under Chromium Toxicity
}

PEREIRA, P.F. ${ }^{2}$, ANTUNES, F. ${ }^{3}$, BRAGA, V.F. ${ }^{4}$, RESENDE, C.F. ${ }^{5}$, RIBEIRO, C. ${ }^{6}$ e PEIXOTO, P.H.P. ${ }^{7}$

\begin{abstract}
RESUMO - Devido à intensa utilização industrial, o cromo é considerado um importante poluente ambiental. O presente trabalho objetivou determinar os teores de pigmentos hidro e lipossolúveis em plantas de salvínia expostas a concentrações crescentes de $\mathrm{Cr}$, visando estabelecer parâmetros bioquímicos para utilização dessa macrófita em programas de biomonitoramento e/ou fitorremediação da poluição causada por esse poluente metálico em ambientes aquáticos. As plantas foram submetidas a concentrações crescentes de $\mathrm{Cr}$ e avaliadas após quatro, seis e dez dias de tratamento. Os resultados dos ensaios permitiram concluir que plantas de salvínia sob condições de estresse por $\mathrm{Cr}$ apresentam reduções nas concentrações das clorofilas $\mathrm{a}, \mathrm{b}$ e total e, em contraste, aumentos nas concentrações de antocianinas totais. Embora a concentração de carotenoides totais não tenha sido alterada em resposta ao $\mathrm{Cr}$, as variações nas concentrações dos demais pigmentos lipossolúveis e dos pigmentos hidrossolúveis observadas nas folhas das plantas de salvínia podem ser utilizadas como parâmetros bioquímicos de biomonitoramento da poluição causada por esse elemento metálico em ambientes aquáticos.
\end{abstract}

Palavras-chave: Salvinia auriculata, biomonitoramento, fitorremediação, macrófitas, pteridófita.

\begin{abstract}
Due to widespread industrial use, chromium is considered a serious environmental pollutant. This study aimed to determine the content of hydrosoluble and liposoluble pigments in salvinia plants exposed to increasing concentrations of $\mathrm{Cr}$, to establish biochemical parameters for the use of macrophyta in pollution bio-monitoring programs and/or phyto-remediation in aquatic environments by this pollutant metal. The plants were exposed to increasing concentrations of $\mathrm{Cr}$ and evaluated after four, six, and ten days of treatment. The test results showed that salvinia plants under stress conditions for $\mathrm{Cr}$ exhibit decreases in the concentrations of chlorophylls $a, b$, and total, and, in contrast, increases in anthocyanin concentrations. Although the concentration of carotenoids has not been altered in response to $\mathrm{Cr}$, the variations in the concentrations of other liposoluble and hydrosoluble pigments found in salvinia plant leaves can be used as biochemical parameters for biomonitoring of pollution caused by this metallic element in aquatic environments.
\end{abstract}

Keywords: Salvinia auriculata, bio-monitoring, phyto-remediation, macrophyte, ferns.

\section{INTRODUÇÃO}

A poluição em ambientes aquáticos, especialmente em reservatórios de água doce, é um dos mais importantes problemas ambientais do mundo moderno (Chandra et al., 2004). Nas últimas décadas, a quantidade de cromo $(\mathrm{Cr})$ liberada nos ecossistemas aquáticos e terrestres aumentou como consequência de diferentes atividades humanas (Saha $\&$ Orvig, 2010). $\mathrm{O}$ cromo pode ser liberado naturalmente no ambiente a partir da decomposição da cromita

Recebido para publicação em 11.7.2011 e aprovado em 9.4.2012.

2 Bióloga, Mestranda em Fisiologia Vegetal, Universidade Federal de Viçosa - DBV/UFV, < pauladafonsecapereira@yahoo.com.br; ${ }^{3}$ Pesquisadora, Dra., do CCA, UFRR, <antunes.flavia@bol.com.br>; ${ }^{4}$ Bióloga, Mestre em Ecologia, PGECOL/UFJF, <viplanta@yahoo.com.br>; ${ }^{5}$ Biólogo, Doutorando em Ecologia, PGECOL/UFJF, <cristianoig2004@hotmail.com>; ${ }^{6}$ Professor, Dr., Dep. de Biologia Geral, DBG/UFV, <cleberson.ribeiro@ufv.br, ${ }^{7}$ Professor, Dr., Dep. de Botânica, UFJF, <paulo.peixoto@ufjf.edu.br>. 
$\left(\mathrm{FeCr}_{2} \mathrm{O}_{4}\right)$ e de outros minerais da crosta terrestre (Augustynowicz et al., 2010). Todavia, a principal fonte de liberação do cromo nos ecossistemas aquáticos está relacionada à aplicação industrial desse metal na metalurgia e nos curtumes (Saha \& Orvig, 2010) e na fabricação de tintas e de corantes (Augustynowicz et al., 2010). Uma vez no ambiente, os estados de oxidação mais estáveis do cromo são o $\mathrm{Cr}$ (III) e o $\mathrm{Cr}$ (VI) (Soares et al., 2008; Gupta et al., 2009), sendo este último o mais tóxico devido à sua elevada mobilidade no solo e em ambientes aquáticos (Sun et al., 2010) e à capacidade de atravessar membranas biológicas, agindo como oxidante, interferindo na absorção de nutrientes e na fotossintese, além de apresentar propriedades carcinogênicas e mutagênicas (Pandey et al., 2010; Nichols et al., 2000).

Em concentrações reduzidas, íons metálicos são essenciais à manutenção e ao desenvolvimento de todos os organismos, uma vez que muitas moléculas, enzimas e reações requerem elementos metálicos (Kucera et al., 2008). Entretanto, quando em quantidades excessivas, os metais podem interferir no metabolismo celular normal e tornando-se tóxicos às plantas e animais (Bertrand et al., 2005; Kucera et al., 2008). Como a maioria das tecnologias utilizadas para remover o excesso de cromo são onerosas e pouco eficientes, a fitorremediação se apresenta como uma alternativa de grande interesse na descontaminação de águas poluídas (Prado et al., 2010). Plantas aquáticas vasculares desempenham papel importante na captura, armazenamento e reciclagem de metais (Chandra et al., 2004), sendo utilizadas na remoção de resíduos sólidos em suspensão, nutrientes, metais pesados, bactérias e outras substâncias orgânicas tóxicas. Dados de fitotoxicidade para plantas aquáticas podem ser utilizados em decisões relacionadas aos riscos ambientais para muitos contaminantes. No caso do cromo, tem sido demonstrado que várias macrófitas aquáticas são capazes de reduzir $\mathrm{Cr}(\mathrm{VI})$ para a forma menos tóxica $\mathrm{Cr}(\mathrm{III})$ durante o processo de biossorção desse metal nas raízes (Prado et al., 2010). Salvinia auriculata é uma pequena pteridófita flutuante de água doce encontrada em regiões tropicais e temperadas. Sob condições favoráveis, essas plantas se disseminam rapidamente por propagação vegetativa
(Baral et al., 2009), colonizando extensas áreas de água em reduzido período de tempo.

O presente trabalho objetivou determinar os teores de pigmentos hidro e lipossolúveis em plantas de salvínia expostas a concentrações crescentes de $\mathrm{Cr}$, visando estabelecer parâmetros bioquímicos específicos que possibilitem a utilização dessa macrófita em programas de biomonitoramento e/ou de fitorremediação da poluição causada por esse poluente metálico em ambientes aquáticos.

\section{MATERIAL E MÉTODOS}

Os experimentos foram conduzidos em sala de crescimento nos meses de outubro e novembro de 2010, utilizando-se plantas aquáticas de Salvinia auriculata mantidas em condições controladas de temperatura (25$30{ }^{\circ} \mathrm{C}$ ), fotoperiodo (16-8 h) e luminosidade $\left(40 \mu\right.$ moles $\left.\mathrm{m}^{-2} \mathrm{~s}^{-1}\right)$. As plantas foram cultivadas em vasos de PVC com 1,5 L de solução nutritiva de Clark (1975), pH 6,0, com 1/3 da força, sendo submetidas ao estresse por cromo [Cr(VI)], na forma de $\mathrm{K}_{2} \mathrm{Cr}_{2} \mathrm{O}_{7}$, nas concentrações de $0 ; 0,5$; 1,$0 ; 1,5$; e 2,0 $\mathrm{mg} \mathrm{L}^{-1}$. As folhas das plantas mantidas nessas condições foram analisadas quanto à composição dos pigmentos lipossolúveis e hidrossolúveis após quatro, seis e dez dias da aplicação do cromo.

As concentrações das clorofilas $a, b$ e totais e dos carotenoides totais foram determinadas pelo método de Lichtenthaler (1987). Amostras de $0,2 \mathrm{~g}$ de tecidos foliares foram maceradas em acetona $80 \%(v / v)$. O extrato foi filtrado e o volume completado para $25 \mathrm{~mL}$. Em seguida, foram realizadas as leituras em espectrofotômetro. A concentração de cada pigmento foi expressa em $\mathrm{mg} \mathrm{g}^{-1}$ de matéria fresca.

A concentração de antocianinas foi determinada segundo Mancinelli (1990). Para a extração, foi utilizado $0,2 \mathrm{~g}$ de tecidos folhiares mantidos em $5 \mathrm{~mL}$ de metanol $\mathrm{HCl} 1 \%$ (v/v) por 24 h, sob refrigeração (Downs \& Siegelman, 1963). O extrato foi filtrado e completado para $10 \mathrm{~mL}$. Em seguida, foi submetido à leitura em espectrofotômetro. A absorvância encontrada foi convertida em moles de antocianina, usando o coeficiente de extinção de $34.300 \mathrm{M}^{-1} \mathrm{~cm}^{-1}$ para a absorção máxima a $530 \mathrm{~nm}$ (Sielgeman $\&$ Hendricks, 1958). A concentração de antocianinas totais foi expressa em $\mu$ moles $\mathrm{g}^{-1} \mathrm{de}$ matéria fresca. 
Os experimentos foram conduzidos em delineamento inteiramente casualizado (DIC) com cinco repetições. Os dados foram submetidos a análises de variância (teste $\mathrm{F}$, $\mathrm{P}<5 \%$ ), e as médias, comparadas pelo teste de Scott-Knott a $5 \%$ de probabilidade.

\section{RESULTADOS E DISCUSSÃO}

As concentrações de clorofila $a$, clorofila $b$ e totais não apresentaram alterações significativas nas folhas das plantas tratadas com o cromo $(\mathrm{Cr})$ nas análises realizadas no quarto e no sexto dia após a aplicação dos tratamentos (Figura 1). Contudo, no décimo dia, observou-se redução significativa nas concentrações de clorofilas $a, b$ e totais. Essa redução foi diretamente proporcional ao aumento das concentrações de $\mathrm{Cr}$ (Figura 1).

Diversos trabalhos relatam variações na concentração de pigmentos em resposta ao estresse por Cr em plantas de Salvinia (Nichols et al., 2000; Shanker et al., 2005; Dhir et al., 2008, 2009), Glycine max e Pistia stratiotes (Ganesh et al., 2008), bem como de Nymphaea spontanea (Choo et al., 2006). Segundo relatos da literatura, o declínio observado nos teores de clorofila pode ser resultante da redução do conteúdo de ferro $(\mathrm{Fe})$ nos tecidos, da redução da eficiência de enzimas envolvidas na biossíntese de clorofilas e/ou da substituição do átomo de $\mathrm{Mg}$ por metais pesados no centro do tetrapirrol das moléculas de clorofila (Dhir et al., 2008, 2009). Shanker et al. (2005) atribuem os decréscimos observados na relação clorofila $a / b$ provocados pela exposição ao $\mathrm{Cr}$ à redução do tamanho da fração periférica do complexo antena. Conforme considerações desses autores, as reduções nos conteúdos de clorofila $b$ pelo $\mathrm{Cr}$ se devem à desestabilização ou à degradação de proteínas da parte periférica do complexo antena; a inativação de enzimas envolvidas na rota de biossintese das clorofilas também poderia contribuir para a redução geral no conteúdo de clorofilas em resposta ao estresse. As reduções nas concentrações de clorofilas também podem estar associadas ao aumento da atividade de clorofilases (Prado et al., 2010) ou, ainda, a alterações ultraestruturais nas membranas (Pettersson et al., 1985). Bassi et al. (1990) sugerem que essa redução seja decorrente do aumento do tamanho dos cloroplastos ou da desestruturação dos tilacoides. Segundo Subrahmanyam (2008), a exposição ao cromo pode induzir alterações na ultraestrutura dos cloroplastos, o que reduz o desenvolvimento do sistema lamelar, originando tilacoides com menos grana.

Em contraste ao observado no presente trabalho, Augustynowicz et al. (2010) mostraram que plantas de Callitriche cophocarpa crescidas em solução com $50 \mu \mathrm{M}$ de $\mathrm{Cr}(\mathrm{VI})$ exibiram atividade fotossintética e morfologia da parte aérea e raízes similares às do controle. Espinoza-Quiñones et al. (2008) também obtiveram resultados semelhantes com plantas de Salvinia auriculata e Eicchornia crassipes, que não exibiram sinais de estresse quando submetidas a concentrações de $\mathrm{Cr}(\mathrm{VI})$ menores que $3,5 \mathrm{mg} \mathrm{L}^{-1}$ e de $\mathrm{Cr}$ (III) menores que $5,0 \mathrm{mg} \mathrm{L}^{-1}$. Em Pistia stratiotes, Mufarrege et al. (2010) verificaram que concentrações de até $7 \mathrm{mg} \mathrm{L}{ }^{-1}$ de $\mathrm{Cr}$ não resultaram em alterações das taxas de crescimento relativo. Todavia, os mecanismos de sintese de clorofila foram alterados pelo metal, embora tenha sido observado que em concentrações reduzidas o $\mathrm{Cr}$ pode aumentar as concentrações de clorofila em plantas de Pistia stratiotes, o que é atribuído ao aumento da disponibilidade de ferro $(\mathrm{Fe})$ nos tecidos das plantas (Mufarrege et al., 2010).

Em nenhuma das análises realizadas, as concentrações de carotenoides apresentaram alterações significativas em resposta ao aumento da concentração de Cr (Figura 2). Resultados similares foram observados por Nichols et al. (2000) em plantas de Salvinia minima tratadas com $\mathrm{Cr}(\mathrm{VI})$ nas concentrações de 1 e $2 \mathrm{mg} \mathrm{L}^{-1}$. Os carotenoides podem atuar como antioxidantes e varredores de espécies reativas de oxigênio geradas sob condições de estresse por metais pesados (Gupta et al., 2009). Além disso, seu acúmulo nos tecidos foliares está relacionado à proteção do aparelho fotossintético contra radiações de alta energia (Demmig-Adams, 1990). Contudo, os resultados encontrados no presente trabalho indicam que esse sistema de fotoproteção não foi significativamente afetado, pelo menos durante o período de avaliação do ensaio.

Em contraste, as concentrações de antocianinas totais foram significativamente maiores na presença de $\mathrm{Cr}$ nas análises realizadas com quatro, seis e dez dias após a imposição dos 


\section{Quarto dia}

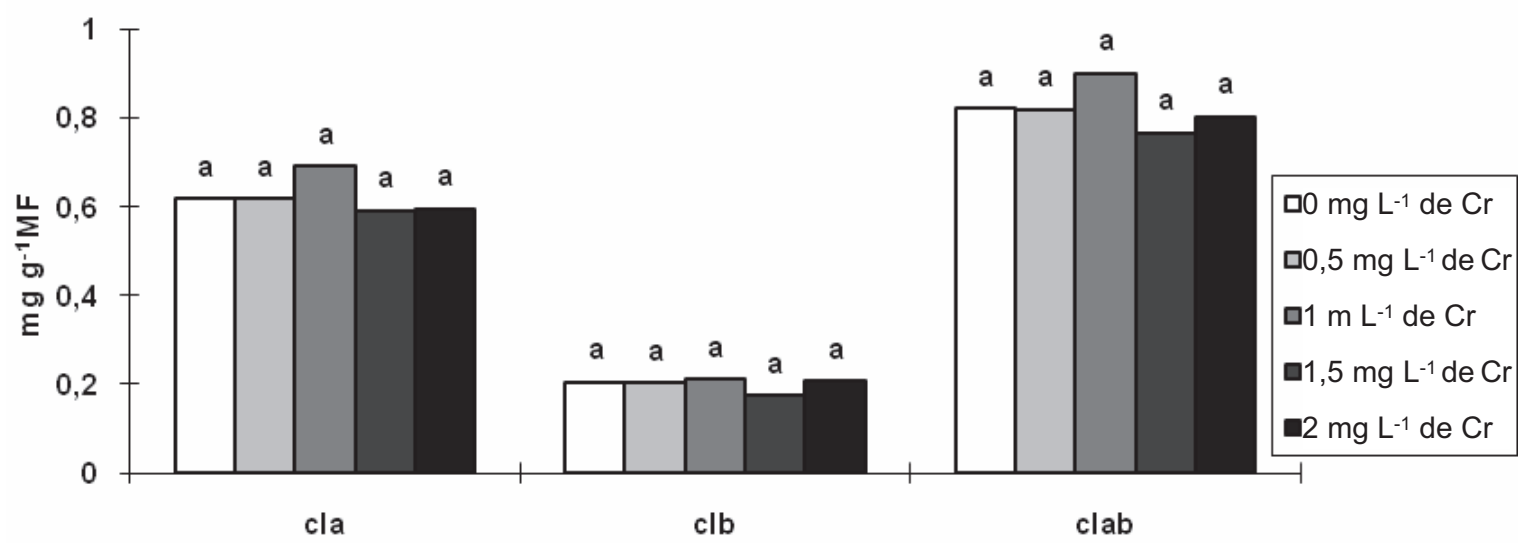

Sexto dia

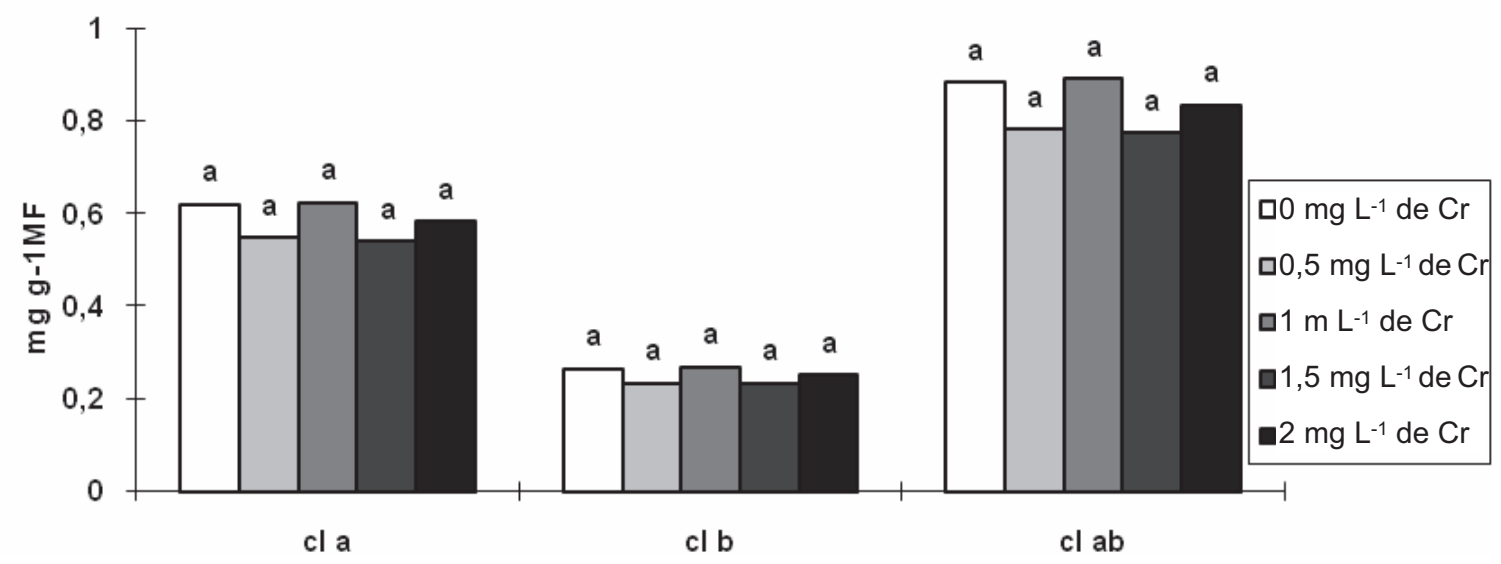

Décimo dia

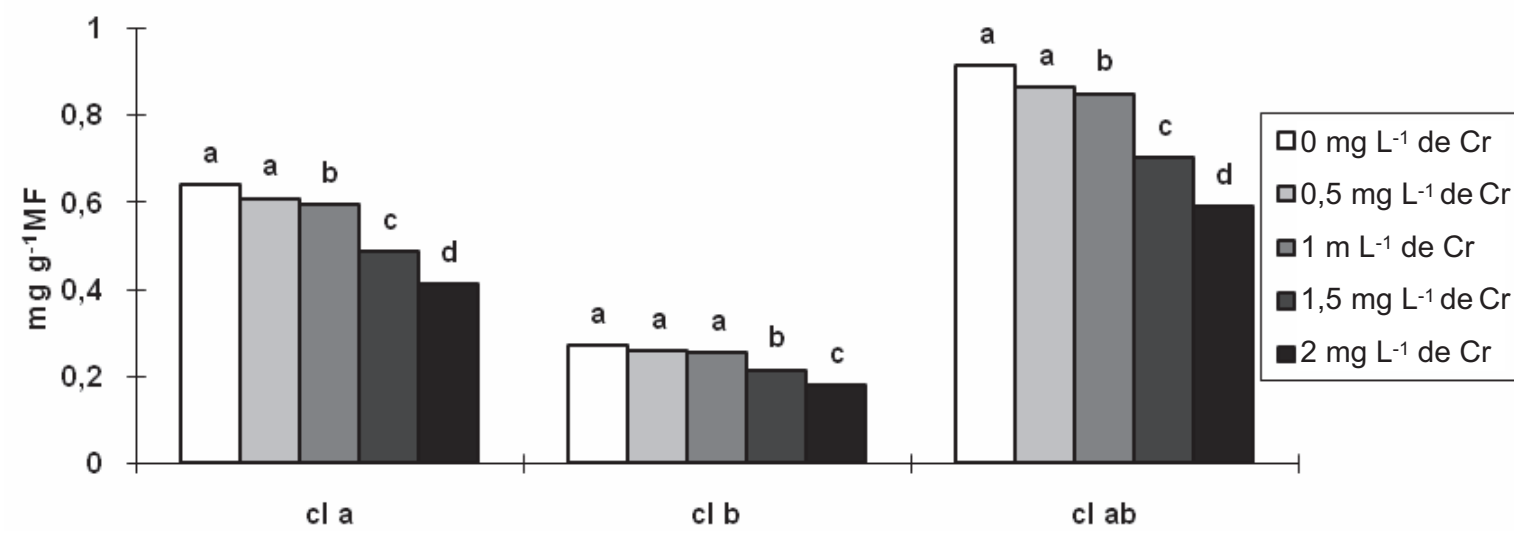

Médias seguidas por mesmas letras (para cada pigmento) não diferem entre si pelo teste de Scott-Knott a $5 \%$ de probabilidade.

Figura 1 - Concentrações de clorofila a (cl a), clorofila b (cl b) e clorofila total (cl ab) em folhas de Salvinia auriculata após quatro, seis e dez dias de aplicação do cromo. 
tratamentos (Figura 3). Trabalhos anteriores reportaram que as antocianinas são produzidas em plantas aquáticas em resposta a diversos fatores de estresse, contribuindo para o sequestro de metais no vacúolo e para a consequente redução da toxicidade citoplasmática (Nichols et al., 2000; Bertrand et al., 2005). No presente estudo, o conteúdo total de

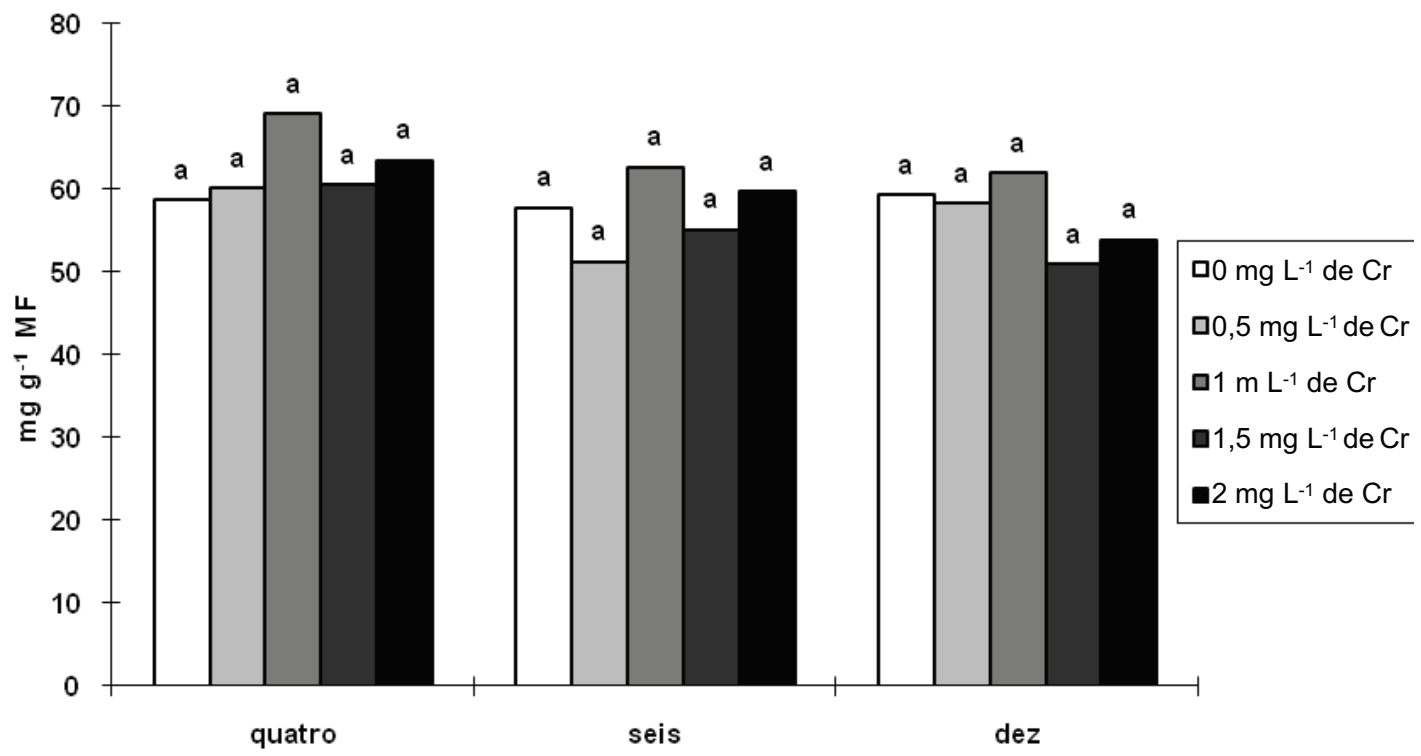

Figura 2 - Concentrações de carotenoides totais em folhas de Salvinia auriculata após quatro, seis e dez dias de aplicação do cromo. Médias seguidas por mesmas letras em cada uma das avaliações não diferem estatisticamente pelo teste de Scott-Knott a $5 \%$ de probabilidade.

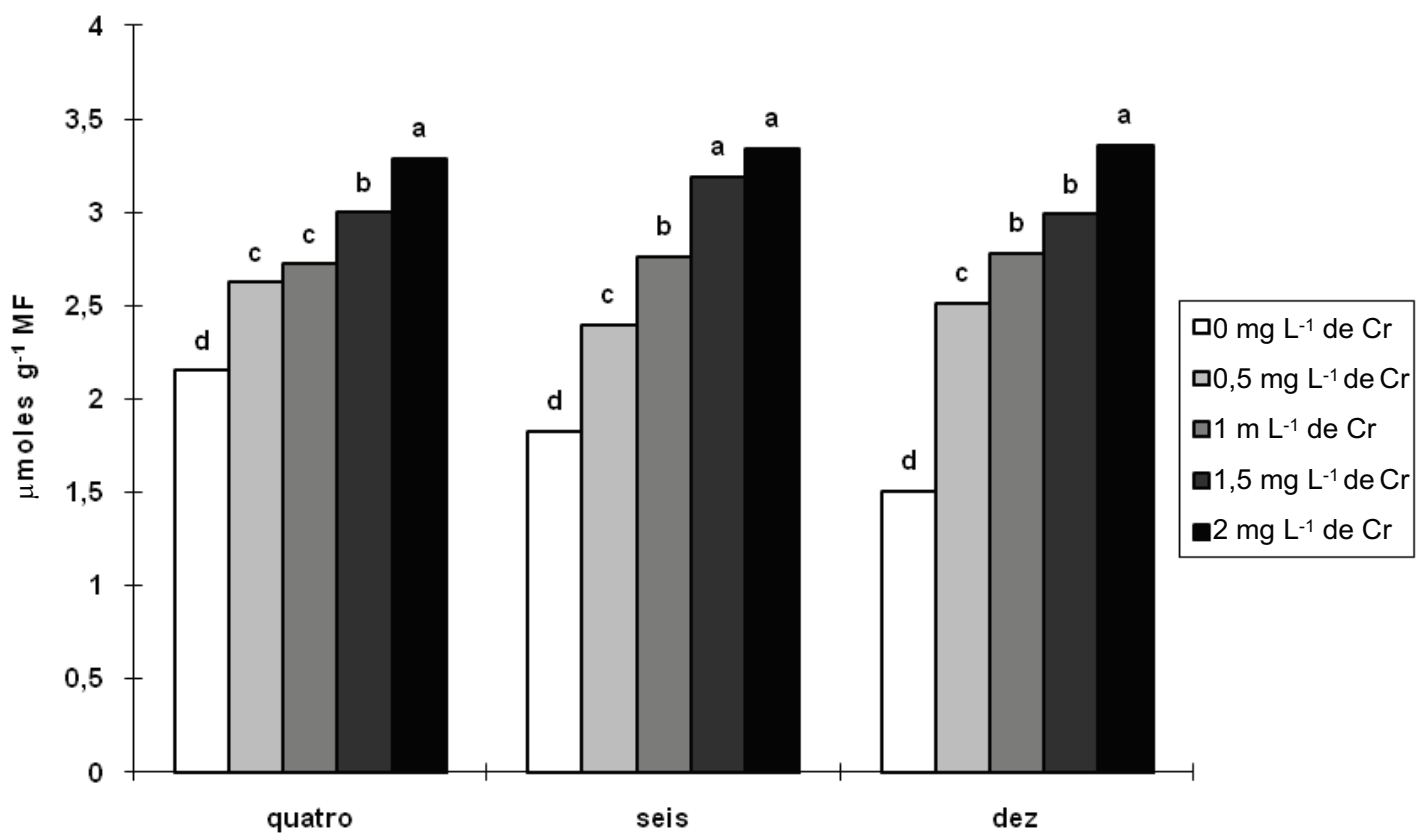

Figura 3 - Concentrações de antocianinas em folhas de Salvinia auriculata após quatro, seis e dez dias de aplicação do cromo. Médias seguidas por mesmas letras em cada uma das avaliações não diferem estatisticamente pelo teste de ScottKnott a $5 \%$ de probabilidade. 
antocianinas aumentou linearmente em resposta ao incremento da concentração do $\mathrm{Cr}$, o que sugere a participação desses flavonoides nas respostas metabólicas das plantas ao estresse causado por esse íon metálico. Um dos efeitos metabólicos associados às antocianinas é a proteção do aparelho fotossintético (Zhanga et al., 2010). Os resultados encontrados no presente trabalho sugerem que, sob condições de toxicidade por $\mathrm{Cr}$, as antocianinas podem ter contribuído mais intensamente na proteção do aparelho fotossintético do que os carotenoides. As antocianinas são antioxidantes muito potentes, atuando em resposta a diferentes estresses oxidativos (Steyn, 2002), o que provavelmente ocorreu neste trabalho em resposta ao estresse causado pelo $\mathrm{Cr}$.

Embora a concentração de carotenoides totais não tenha sido alterada, verificou-se que, sob condições de estresse por $\mathrm{Cr}$, plantas de salvínia apresentam aumentos nas concentrações das clorofilas $a, b$ e totais e, também, nas concentrações de antocianinas totais. Conclui-se, portanto, que as variações nas concentrações dos pigmentos hidro e lipossolúveis em plantas de salvínia podem ser utilizadas como parâmetros bioquímicos no monitoramento da poluição causada pelo $\mathrm{Cr}$ em ambientes aquáticos.

\section{AGRADECIMENTOS}

À Fundação de Amparo à Pesquisa do Estado de Minas Gerais (FAPEMIG) pelo apoio financeiro na execução e publicação deste trabalho.

\section{LITERATURA CITADA}

AUGUSTYNOWICZ, J. et al. Chromium(VI) bioremediation by aquatic macrophyte Callitriche cophocarpa Sendtn.

Chemosphere, v. 79, n. 11, p. 1077-1083, 2010.

BARAL, S. S. et al. Removal of Cr(VI) by thermally activated weed Salvinia cucullata in a fixed-bed column. J. Hazard Mater., v. 161, n. 2-3, p. 1427-1435, 2009.

BASSI, M.; CORRADI, G.; RICCI, A. Effects of chromium (VI) on two freshwater plants, Lemna minor and Pistia stratiotes; biochemical and physiological observations. Cytobios, v. 62, n. 249, p.101-109, 1990.

BERTRAND, M.; POIRIER, I. Photosynthetic organisms and excess of metals. Photosynthetica, v. 43, n. 3, p. 345-353, 2005.

Planta Daninha, Viçosa-MG, v. 30, n. 4, p. 397-703, 2012
CHANDRA, P.; KULSHRESHTHA, K. Chromium accumulation and toxicity in aquatic vascular plants. Bot. Rev., v. 70, n. 3, p. 313-327, 2004.

CHOO, T. P. et al. Accumulation of chromium (VI) from aqueous solutions using water lilies (Nymphaea spontanea). Chemosphere, v. 62, n. 6, p. 961-967, 2006.

CLARK, R. B. Characterization of phosphatase of intact maize roots. J. Agric. Food Chem., v. 23, n. 3, p. 458-460, 1975.

DEMMIG-ADAMS, B. Carotenoids and photoprotection in plants: a role for the xanthophylls zeaxanthin. BBBABioenerg., v. 1020, n. 1, p. 1-24, 1990.

DHIR, B.; SHARMILA, P.; SARADHI, P. P. Photosynthetic performance of Salvinia natans exposed to chromium and zinc rich wastewater. Braz. J. Plant Physiol., v. 20, n. 1, p. 61-70, 2008.

DHIR, B. et al. Physiological and antioxidant responses of Salvinia natans exposed to chromium-rich wastewater.

Ecotox. Environ. Safe., v. 72, n. 6, p. 1790-1797, 2009.

DOWNS, R. J.; SIEGELMAN, H. W. Photocontrol of anthocyanin synthesis in milo seedlings. Plant Physiol., v. 38 , n. 1 , p. $25-30,1963$.

ESPINOZA-QUIÑONES, F. R. et al. Chromium ions phytoaccumulation by three floating aquatic macrophytes from a nutrient medium. World J. Microbiol. Biot., v. 24, n. 12 , p. 3063-3070, 2008.

GANESH, K. S. et al. Chromium stress induced alterations in biochemical and enzyme metabolism in aquatic and terrestrial plants. Colloids Surfaces B: Biointerf., v. 63, n. 2, p. 159-163, 2008.

GUPTA, S.; SRIVASTAVA, S.; SARADHI, P. P. Chromium increases photosystem 2 activity in Brassica juncea. Biol. Plant., v. 53, n. 1, p. 100-104, 2009.

KUÈERA, T.; HORÁKOVÁ, H.; ŠONSKÁ, A. Toxic metal ions in photoautotrophic organisms. Photosynthetica, v. 46, n. 4, p. 481-489, 2008.

LICHTENTHALER, H. K. Clorophylls and carotenoids: pigments of photosynthetic biomembranes. Methods Enzimol., v. 148, p. 350-382, 1987.

MANCINELLI, A. L. Interaction between light quality and light quantity in photoregulation of anthocyanin production. Plant Physiol., v. 92, n. 4, p. 1191-1195, 1990.

MUFARREGE, M. M.; HADAD, H. R.; MAINE, M. A. Response of Pistia stratiotes to heavy metals $(\mathrm{Cr}, \mathrm{Ni}$, and $\mathrm{Zn})$ and Phosphorous. Archi. Environ. Cont. Toxicol., v. 58, n. 1, p. 53-61, 2010. 
NICHOLS, P. B.; COUCH, J. D.; AL-HAMDANI, S. H. Selected physiological responses of Salvinia minima to different chromium concentrations. Aquat. Bot., v. 68, n. 4, p. 313-319, 2000.

PANDEY, V.; DIXIT, V.; SHYAM, R. Chromium effect on ROS generation and detoxification in pea (Pisum sativum) leaf chloroplasts. Protoplasma, v. 236, n. 1-4, p. 85-95, 2010.

PETTERSSON, A. L.; HAELLBOM, L.; BERGMAN, B. Physiological and structural responses of the cyanobacterium Anabaena cylindrica to aluminum. Physiol. Plant., v. 63, n. 2, p. 153-158, 1985.

PRADO, C. et al. Uptake of chromium by Salvinia minima: effect on plant growth, leaf respiration and carbohydrate metabolism. J. Hazard. Mater., v. 177, n. 1-3, p. 546-553, 2010 .

SAHA, B.; ORVIG, C. Biosorbents for hexavalent chromium elimination from industrial and municipal effluents. Coordin. Chem. Rev., v. 254, n. 23-24, p. 2959-2972, 2010.

SHANKER, A. K. et al. Chromium toxicity in plants. Environ. Int., v. 31, n. 5, p. 739- 753, 2005.
SIEGELMAN, H. W.; HENDRICKS, S. B. Photocontrol of anthocyanin synthesis in apple skin. Plant Physiol., v. 33, n. 3, p. 185-190, 1958.

SOARES, D. C. F. et al. Salvinia auriculata: aquatic bioindicator studied by instrumental neutron activation analysis (INAA). Appl. Radiat. Isotopes, v. 66, n. 5, p. 561-564, 2008.

STEYN, W. J. et al. Anthocyanins in vegetative tissues: a proposed unified function in photoprotection. New Phytol., v. 155, n. 3, p. 349-361, 2002.

SUBRAHMANYAM, D. Effects of chromium toxicity on leaf photosynthetic characteristics and oxidative changes in wheat (Triticum aestivum L.). Photosynthetica, v. 46, n. 3, p. 339-345, 2008.

SUN, X-F. et al. Sorption and detoxification of chromium(VI) by aerobic granules functionalized with polyethylenimine.

Water Res., v. 44, n. 8, p. 2517-2524, 2010.

ZHANGA, K-M. et al. Photoprotective roles of anthocyanins in Begonia semperflorens. Plant Sci., v. 179, n. 3, p. 202-208, 2010. 